\title{
Catch-up alveolar development into adulthood: also in those born prematurely?
}

\author{
Peter J.F.M. Merkus
}

Affiliation: Dept of Paediatrics, Division of Respiratory Medicine and Allergy, Amalia Children's Hospital, Radboud University Nijmegen Medical Centre, Nijmegen, The Netherlands.

Correspondence: Peter J.F.M. Merkus, Dept of Paediatrics, Division of Respiratory Medicine and Allergy, Amalia Children's Hospital, Radboud University Nijmegen Medical Centre, P0 Box 9101, 6500 HB, The Netherlands.

E-mail: Peter.Merkusaradboudumc.nl

0 @ERSpublications

The window of possible catch-up alveolarisation seems quite large: good news for the young and the old http://ow.ly/WNPIV

The way the human lung grows post-natally has intrigued many of us and many of our predecessors, and still does. Originally exclusively based on post mortem findings, it was concluded that a large variability in number of alveoli exists between humans and that this wide scatter is present not only at birth but also in childhood $[1,2]$. From these cross-sectional anatomical studies, it could not be inferred whether catch-up (increase of alveolar numbers) is possible, whether interindividual differences in longitudinal growth patterns exist and when physiological alveolar multiplication ends. Classically, many authors assumed that new alveoli are formed up to at least 8 years of age $[3,4]$ but from the largest morphometric study, it was concluded that the number of alveoli in both sexes increased little or not at all after the age of 2 years [2]. The hypothesis was that thereafter, further lung growth consists of dimensional growth of alveoli only. Similar growth patterns were assumed to exist in post-pneumectomy compensatory lung growth, with very little indication that multiplication occurs in adolescence or thereafter [5]. Obviously, to study the alveolar increase during growth, other techniques should be used.

Methods suitable to study longitudinal growth of airways and airspaces are the techniques that assess respiratory function in health $[6-8]$ and disease $[9,10]$ or the techniques revealing lung structure $[11,12]$. Although several studies have actually attempted to assess growth of lungs and airways during childhood and/or adolescence, it is difficult to draw solid conclusions because of significant limitations in the techniques themselves or in the interpretations of results.

Furthermore, the assessment of growth of the lung parenchyma and of alveolarisation is complicated by physiological changes: during childhood, the compliance of the chest wall decreases while that of the lung parenchyma increases. This results in a relative under-inflation during early childhood, and a relative overinflation in adolescence and adulthood $[13,14]$. This also explains the relative decrease in closing volume with age [13] and can be seen as an improvement of mixing efficiency of the lung during growth. So, it is a natural phenomenon that the lung is relatively under-distended in infants and preschool children, and relatively over-distended from childhood to adulthood: hence, one could conclude that the physiological aeriation of the lungs and, consequently, also alveolar size, is age dependent. Clearly, this has consequences for structural and functional assessments of the lungs.

Besides that, interpreting respiratory function studies is complex and not straightforward, because not all variables are controlled for or known, and because the sensitivity of the techniques may be low. Most respiratory function studies reflect (mainly central) airway function, and not actual function of the peripheral airways or lung parenchyma. When trying to elucidate specific tests that are supposed to reflect

Received: Jan 032016 | Accepted: Jan 032016

Conflict of interest: None declared.

Copyright OERS 2016 
function of the parenchyma or the small airways, we are also faced with relevant shortcomings. For instance, a decrease of lung clearance index may reflect improvement of the ventilation inhomogeneity of the lung or increased homogeneity of ventilation due to complete closure of poorly ventilated lung compartments, and when diffusing capacity of the lung is maintained, this does not rule out that significant structural damage and decline of forced expiratory volume in $1 \mathrm{~s}$ (FEV1) has occurred, accompanied by redistribution of pulmonary perfusion [15].

Most imaging studies potentially suffer from a limitation of the resolution, complicating estimates of (changes in) alveolar size. However, a major improvement in resolution of alveolar size was established using hyperpolarized, ventilation-weighted helium magnetic resonance imaging $\left({ }^{3} \mathrm{He}-\mathrm{MRI}\right)$. This technique is known for a far better resolution such that the alveolar sizes are much better distinguished. ${ }^{3} \mathrm{He}-\mathrm{MRI}$ directly visualises regional ventilation heterogeneity and is sensitive to changes of ventilation. The estimate of the alveolar dimensions is based on the calculated apparent diffusion coefficient (ADC), using the capability of ${ }^{3} \mathrm{He}$-MRI to quantify Brownian gas diffusion [16]. This makes it an excellent tool to study alveolarisation of the lungs. Using ${ }^{3} \mathrm{He}-\mathrm{MRI}$, it was concluded that alveolarisation increases beyond early childhood in to adulthood [16], that compensatory lung growth following pneumectomy in adulthood is explained not only by dimensional increase of existing alveoli but also by development of new alveoli [17], and that some degree of catch-up growth of alveoli is possible following extreme prematurity [18]. These spectacular conclusions, however, are only valid as long as the assumption is correct that, based on ADC, we can infer the spatial dimensions of the alveolar airspaces.

Given the limitations of each technique, should we conclude that proper longitudinal studies of the quantity and quality of alveoli are not yet feasible or even impossible? Not necessarily so. When we find indications from multiple and different angles that the lungs can continue alveolarisation during a large part of life, under different conditions, this makes it all the more likely that indeed the lung is a relatively plastic organ, and this will also have clinical consequences. This is where the article by Yammine et al. [19], in a recent issue of the European Respiratory Journal, comes in. In an elegant way, they studied Sacin, focusing on the respiratory function of the peripheral lung, and provide additional suggestive evidence from a functional study that catch-up alveolarisation is possible, at least until school age, in children born prematurely.

It is especially interesting that in subjects born prematurely, such a catch up of alveolarisation would exist. It has been well documented that prematurity as such is a major disruptor of normal lung growth. The premature birth and the subsequent post-natal exposure to $21 \%$ oxygen or more cause a developmental arrest of lung growth, even in the absence of artificial ventilation or neonatal respiratory distress [20]. This has been extensively studied in animal experiments and has been explained by diminished angiogenesis that is the growth limiting factor of alveolarisation [21]. This results in an underdevelopment of the pulmonary vasculature and in fewer but larger alveoli, with fewer alveolar attachments, and increased airway resistance and work of breathing [22], and is associated with diminished level and abnormal development of lung function in former pre-terms with or without a history of artificial ventilation [23-25]. These studies of early lung function following prematurity do not demonstrate any catch-up of airway function in the first year and even a relative deterioration compared to normals. In addition, in later life, reports suggest that airway function in these patients tracks, without signs of any catch-up growth $[26,27]$.

So apparently, the lung has some capacity to organise a catch-up growth of alveolarisation over the years and that is very good news [28]. This growth acceleration was labelled as dysanaptic growth by YAMmINE et al. [19] and indeed, growth of airway function and parenchymal growth are probably out of phase during a certain time window. The term dysanapsis, defined as unequal growth of the lung, was first introduced by MEAD [6], who observed marked variability of the relationship between forced expiratory flows and lung volumes that could not be explained by differences in elastic recoil of the lung. These findings, suggestive of differential growth patterns between airway function and that of the parenchyma, were confirmed by others [7,8] and actually may simply reflect mechanisms supporting the Barker hypothesis that was launched many years later [29], which could also explain the large interindividual variability of alveolar numbers [1-4]. Hence, differential growth patterns of the lung parenchyma relative to airway calibre exist and may have many causes, prematurity being one of them. However, it remains a miracle that alveolar growth can accelerate in these patients because in early post-natal life, it can be arrested due to the lack of adequate pulmonary angiogenesis [20,21]. Further studies are clearly needed to explain that phenomenon.

So, the lung seems to possess a greater capacity for regeneration than previously recognised [30] and this catch-up of alveolarisation occurs independently of the growth of FEV1. Therefore, this catch up of alveolar growth in formerly pre-term infants may not indicate a better prognosis. One of the main risk factors and 
causes of respiratory symptoms, respiratory disease or hospital admissions in this group will remain diminished airway function and increased work of breathing. As long as airway function does not improve, any increase in or normalisation of alveolar numbers is not necessarily associated with a better prognosis in formerly pre-term patients: this is supported the study by WALTER et al. [31], who studied a group of about 150000 children in which they found a relationship between birth weight and cumulative hospital admissions. When using birth weight as a proxy for gestational age, it demonstrated that children with a birth weight $<1250 \mathrm{~g}$ had a persistent increase of cumulative incidence of hospital admissions, from infancy into early adulthood. This implies that these subjects have a condition that they do not "grow out of".

So, in spite of the possible catch-up of alveolarisation in the pre-term category, morbidity remains relatively high and does not level off or converge to the patterns observed in those who had a normal birth weight. Then, what else do these new insights bring our patients, and us? We can only speculate on the potential of future therapeutic strategies that stimulate alveolarisation, but particularly those patients suffering from parenchymal disease would be candidate patients, and this may take many years before realisation. Maybe the first and most clear therapeutic consequence of these insights is that timing of lung surgery is less crucial than we previously thought and that a delay of surgery in growing subjects does not necessarily have a detrimental effect on the quality of the parenchyma. In addition, in adult patients, the compensatory growth will yield an addition of normal alveoli. Hence, timing of elective surgery can be delayed in, for example, some young children with congenital malformations of the lungs or airways. Also, the window of possible catch-up alveolarisation actually seems quite large, so this is not only good news for the young but also for the old.

\section{References}

1 Hislop AA, Wigglesworth JS, Desai R. Alveolar development in the human fetus and infant. Early Hum Dev 1986; 13: $1-11$.

Thurlbeck WM. Postnatal human lung growth. Thorax 1982; 37: 564-571.

Dunnill MS. The problem of lung growth. Thorax 1982; 37: 561-563.

Reid LM. Lung growth in health and disease. Br J Dis Chest 1984; 78: 113-134.

Cagle PT, Thurlbeck WM. Postpneumonectomy compensatory lung growth. Am Rev Respir Dis 1988; 138 : $1314-1326$.

6 Mead J. Dysanapsis in normal lungs assessed by the relationship between maximal flow, static recoil, and vital capacity. Am Rev Respir Dis 1980; 121: 339-342.

7 Merkus PJ, Borsboom GJ, Van Pelt W, et al. Growth of airways and air spaces in teenagers is related to sex but not to symptoms. J Appl Physiol (1985) 1993; 75: 2045-2053.

8 Martin TR, Feldman HA, Fredberg JJ, et al. Relationship between maximal expiratory flows and lung volumes in growing humans. J Appl Physiol (1985) 1988; 65: 822-828.

9 Merkus PJ, van Pelt W, van Houwelingen JC, et al. Inhaled corticosteroids and growth of airway function in asthmatic children. Eur Respir J 2004; 23: 861-868.

10 Merkus PJ, van Essen-Zandvliet EE, Kouwenberg JM, et al. Large lungs after childhood asthma. A case-control study. Am Rev Respir Dis 1993; 148: 1484-1489.

11 de Jong PA, Long FR, Wong JC, et al. Computed tomographic estimation of lung dimensions throughout the growth period. Eur Respir J 2006; 27: 261-267.

12 de Jong PA, Nakano Y, Lequin MH, et al. Estimation of lung growth using computed tomography. Eur Respir J 2003; 22: 235-238.

13 Mansell AL, Bryan AC, Levison H. Relationship of lung recoil to lung volume and maximum expiratory flow in normal children. J Appl Physiol Respir Environ Exerc Physiol 1977; 42: 817-823.

14 Cooper DM, Mellins RB, Mansell AL. Changes in distribution of ventilation with lung growth. J Appl Physiol Respir Environ Exerc Physiol 1981; 51: 699-705.

15 Merkus PJ, Govaere ES, Hop WH, et al. Preserved diffusion capacity in children with cystic fibrosis. Pediatr Pulmonol 2004; 37: 56-60.

16 Narayanan M, Owers-Bradley J, Beardsmore CS, et al. Alveolarization continues during childhood and adolescence: new evidence from helium-3 magnetic resonance. Am J Respir Crit Care Med 2012; 185: 186-191.

17 Butler JP, Loring SH, Patz S, et al. Evidence for adult lung growth in humans. N Engl J Med 2012; 367: 244-247.

18 Narayanan M, Beardsmore CS, Owers-Bradley J, et al. Catch-up alveolarization in ex-preterm children: evidence from ${ }^{3} \mathrm{He}$ magnetic resonance. Am J Respir Crit Care Med 2013; 187: 1104-1109.

19 Yammine S, Schmidt A, Sutter O, et al. Functional evidence for continued alveolarisation in former preterms at school age? Eur Respir J 2016; 47: 147-155.

20 van Tuyl M, Liu J, Wang J, et al. Role of oxygen and vascular development in epithelial branching morphogenesis of the developing mouse lung. Am J Physiol Lung Cell Mol Physiol 2005; 288: L167-L178.

21 Jakkula M, Le Cras TD, Gebb S, et al. Inhibition of angiogenesis decreases alveolarization in the developing rat lung. Am J Physiol Lung Cell Mol Physiol 2000; 279: L600-L607.

22 Baraldi E, Filippone M. Chronic lung disease after premature birth. N Engl J Med 2007; 357: 1946-1955.

23 Hofhuis W, Huysman MW, van der Wiel EC, et al. Worsening of V'maxFRC in infants with chronic lung disease in the first year of life: a more favorable outcome after high-frequency oscillation ventilation. Am J Respir Crit Care Med 2002; 166: 1539-1543.

24 Gappa M, Stocks J, Merkus P. Lung growth and development after preterm birth: further evidence. Am J Respir Crit Care Med 2003; 168: 399.

25 Hoo AF, Gupta A, Lum S, et al. Impact of ethnicity and extreme prematurity on infant pulmonary function. Pediatr Pulmonol 2014; 49: 679-687. 
26 Filippone M, Sartor M, Zacchello F, et al. Flow limitation in infants with bronchopulmonary dysplasia and respiratory function at school age. Lancet 2003; 361: 753-754.

27 Filippone M, Bonetto G, Cherubin E, et al. Childhood course of lung function in survivors of bronchopulmonary dysplasia. JAMA 2009; 302: 1418-1420.

28 Jobe AH. Good news for lung repair in preterm infants. Am J Respir Crit Care Med 2013; 187: 1043-1044.

29 Barker DJ, Osmond C. Infant mortality, childhood nutrition, and ischaemic heart disease in England and Wales. Lancet 1986; 1: 1077-1081.

30 Baker CD, Alvira CM. Disrupted lung development and bronchopulmonary dysplasia: opportunities for lung repair and regeneration. Curr Opin Pediatr 2014; 26: 306-314.

31 Walter EC, Koepsell TD, Chien JW. Low birth weight and respiratoryhospitalizations in adolescence. Pediatr Pulmonol 2011; 46: 473-482. 